\title{
Enhancement of BLIS production by Pediococcus acidilactici kp10 in optimized fermentation conditions using an artificial neural network
}

\begin{abstract}
The present study was aimed at enhancing the production of bacteriocin-like inhibitory substance (BLIS) produced by Pediococcus acidilactici Kp10 through optimizing the fermentation parameters. M17 was chosen in preliminary study as a culture medium because BLIS production was nine times higher (1427.7 AU mL-1) compared to that produced by MRS (160 AU mL-1). The fermentation parameters such as temperature, inoculum size, buffer strength, concentration of tween 80 and agitation speed were screened using two level half-factorial design. BLIS production is influenced by three most significant factors identified as temperature, inoculum size and agitation speed, which were further optimized using an artificial neural network (ANN). ANN predicted that a maximum activity of 5262.64 AU mL-1 would be obtained at optimum conditions of $120 \mathrm{rpm}, 3 \%$ and $28.5{ }^{\circ} \mathrm{C}$. The observed BLIS activity at the predicted optimum levels of the tested variables in ANN was 5118.5 AU mL-1, which was close to the predicted BLIS activity. Increased BLIS activity in the final solution, which resulted from the optimized process, would reduce downstream steps such as concentrating the product during purification.
\end{abstract}

Keyword: BLIS production; Pediococcus acidilactici kp10; Fermentation conditions; Artificial neural network 